UMTG-278

\title{
Singular solutions, repeated roots and completeness for higher-spin chains
}

\author{
Wenrui Hao 1, Rafael I. Nepomechie 2 and Andrew J. Sommese 3
}

\begin{abstract}
We investigate the completeness of the solutions of the Bethe equations for the integrable spin- $s$ isotropic (XXX) spin chain with periodic boundary conditions. Solutions containing the exact string $i s, i(s-1), \ldots,-i(s-1),-i s$ are singular. For $s>1 / 2$, there exist also "strange" solutions with repeated roots, which nevertheless are physical (i.e., correspond to eigenstates of the Hamiltonian). We derive conditions for the singular solutions and the solutions with repeated roots to be physical. We formulate a conjecture for the number of solutions with pairwise distinct roots in terms of the numbers of singular and strange solutions. Using homotopy continuation, we solve the Bethe equations numerically for $s=1$ and $s=3 / 2$ up to 8 sites, and find some support for the conjecture. We also exhibit several examples of strange solutions.
\end{abstract}

\footnotetext{
${ }^{1}$ Mathematical Biosciences Institute, The Ohio State University, 1735 Neil Avenue, Columbus, OH 43210 USA, hao.50@osu.edu

2 Physics Department, P.O. Box 248046, University of Miami, Coral Gables, FL 33124 USA, nepomechie@physics.miami.edu

${ }^{3}$ Department of Applied and Computational Mathematics and Statistics, University of Notre Dame, 153 Hurley Hall, IN 46556 USA, sommese@nd.edu
} 


\section{Introduction}

The spin-1/2 isotropic Heisenberg quantum spin chain, which is well known to be integrable and therefore solvable by Bethe ansatz (see e.g. [1]), has an integrable spin- $s$ generalization $(s=1 / 2,1,3 / 2, \ldots)[2,6,4,5,6]$. Since there is a spin- $s$ spin at each of the $N$ sites, the Hilbert space is $\left(\mathcal{C}^{2 s+1}\right)^{\otimes N}$. The Hamiltonian is given (up to an additive constant) by [1, 6]

$$
H_{s}=\sum_{n=1}^{N} Q_{2 s}\left(\vec{s}_{n} \cdot \vec{s}_{n+1}\right), \quad \vec{s}_{N+1} \equiv \vec{s}_{1},
$$

where $\vec{s}$ are the spin-s generators of $s u(2), \vec{s}_{n}$ denotes the spin operators at site $n, Q_{2 s}(x)$ is a polynomial of degree $2 s$ given by

$$
Q_{2 s}(x)=\sum_{j=1}^{2 s} h(j) \prod_{\substack{l \neq j \\ l=0}}^{2 s} \frac{x-x_{l}}{x_{j}-x_{l}}, \quad x_{l}=\frac{1}{2} l(l+1)-s(s+1),
$$

and $h(j)$ is the $j^{\text {th }}$ harmonic number,

$$
h(j)=\sum_{k=1}^{j} \frac{1}{k} .
$$

For example, for $s=1, Q_{1}(x)=\frac{1}{4}\left(x-x^{2}\right)+\frac{3}{2}$. The eigenvalues of the Hamiltonian (1.1) are given by [1, [5, 6]

$$
E_{s}=-\sum_{k=1}^{M} \frac{s}{\lambda_{k}^{2}+s^{2}}
$$

where $\left\{\lambda_{1}, \ldots, \lambda_{M}\right\}$ satisfy the spin- $s$ Bethe equations

$$
\begin{array}{r}
\left(\lambda_{k}+i s\right)^{N} \prod_{\substack{j \neq k \\
j=1}}^{M}\left(\lambda_{k}-\lambda_{j}-i\right)=\left(\lambda_{k}-i s\right)^{N} \prod_{\substack{j \neq k \\
j=1}}^{M}\left(\lambda_{k}-\lambda_{j}+i\right), \\
k=1,2, \ldots, M, \quad M=0,1, \ldots, s N .
\end{array}
$$

The corresponding Bethe states are given by

$$
\left|\lambda_{1}, \ldots, \lambda_{M}\right\rangle=\prod_{j=1}^{M} B\left(\lambda_{j}\right)|0\rangle, \quad|0\rangle=\left(\begin{array}{c}
1 \\
0 \\
\vdots \\
0
\end{array}\right)^{\otimes N}
$$

where the creation operator $B(\lambda)$ is defined in Appendix $\mathrm{A}$, and $|0\rangle$ is the reference state with all spins "up" (eigenstates of $s^{z}$ with eigenvalue $s$ ). These states are eigenvectors of the Hamiltonian (1.1) with eigenvalue (1.4) as well as $s u(2)$ highest-weight states

$$
S^{+}\left|\lambda_{1}, \ldots, \lambda_{M}\right\rangle=0, \quad S^{ \pm}=S^{x} \pm i S^{y}, \quad \vec{S}=\sum_{n=1}^{N} \vec{s}_{n}
$$


and are therefore eigenstates of $\vec{S}^{2}$ and $S^{z}$ with corresponding eigenvalues $S(S+1)$ and $m$ (respectively) with

$$
S=m=s N-M
$$

The states with lower values of $m$ can be obtained by acting on the Bethe states (1.6) with the spin lowering operator $S^{-}$.

For $s>1 / 2$, the models (1.1) are perhaps not realizable experimentally. Nevertheless, these models have interesting connections to the level $k=2 s$ Wess-Zumino-Witten model and RSOS models (see e.g. [1, 7, 8] and references therein).

A still-unanswered question is whether the Bethe ansatz solution is "complete"; i.e., whether the Bethe equations (1.5) have the correct number of solutions to account for all $(2 s+1)^{N}$ eigenstates of the Hamiltonian.

For the $s=1 / 2$ chain, this problem was already discussed by Bethe in his original paper [9], and was subsequently further investigated by others (see e.g. [1, 10, 11, 12, 13, 14, 15, [16]). Part of the difficulty of this problem is due to the existence of so-called singular (or exceptional) solutions of the Bethe equations, i.e. solutions that contain $\pm i / 2$, and which do not necessarily correspond to eigenstates of the Hamiltonian (see e.g. [17, 18, 19, 20, 21, 22, [23, 24, 25]). Another difficulty is that the possibility of repeated Bethe roots has not been ruled out.

We recently formulated, for this $s=1 / 2$ case, a precise conjecture [26] for the number of solutions of the Bethe equations with pairwise distinct roots, in terms of numbers of singular solutions. Using homotopy continuation methods (see e.g. [27, 28] and references therein), we solved the Bethe equations numerically for values of $N$ up to 14, and found perfect agreement with this conjecture. Its meaning is that the Bethe equations generally have too many solutions with pairwise distinct roots; but after discarding the unphysical singular solutions, there remain exactly the right number of solutions to account for all $2^{N}$ eigenstates of the Hamiltonian.

Our goal in the present work is to extend our $s=1 / 2$ results [26] to arbitrary values of $s$. We find that the notion of singular solution neatly generalizes to the spin- $s$ case: a solution is singular if it contains an exact string of length $2 s+1$ centered at the origin, i.e.

$$
\{i s, i(s-1), \ldots,-i(s-1),-i s\} \text {. }
$$

However, in contrast with the $s=1 / 2$ case, we find that for $s>1 / 2$ there also exist "strange" solutions: solutions of the Bethe equations that have repeated roots, which nevertheless produce eigenstates of the Hamiltonian. Consequently, checking completeness is significantly more complicated.

The outline of this paper is as follows. In Section 2 we formulate a conjecture for the number of solutions of the Bethe equations (1.5) with pairwise distinct roots in terms of the numbers of singular and strange solutions. In Section 3 we present some numerical support for this conjecture for the cases $s=1$ and $s=3 / 2$. We also exhibit several examples of strange solutions. Our analysis relies on important conditions for singular and strange solutions to be physical, the derivations of which are presented in Appendices $\mathrm{A}$ and $\mathrm{B}$, respectively. A brief discussion of our results is given in Section 4 . 


\section{The completeness conjecture}

As in the $s=1 / 2$ case [26], we denote by $\mathcal{N}(N, M)$ the number of solutions of the Bethe equations (1.5) for given values of $N$ and $M$ with pairwise distinct roots (i.e., $\lambda_{j} \neq \lambda_{k}$ for $j \neq k$ ). We always count solutions up to permutations of the roots: if $\left\{\lambda_{1}, \ldots, \lambda_{M}\right\}$ is a solution, then any permutation of these $\lambda_{k}$ 's is not counted as a separate solution.

A naive conjecture for $\mathcal{N}(N, M)$ can be obtained on the basis of the Clebsch-Gordan theorem. Indeed, the $N$-fold tensor product of spin- $s$ representations decomposes into a direct sum of (irreducible) spin- $S$ representations,

$$
\underbrace{\mathbf{s} \otimes \cdots \otimes \mathbf{s}}_{N}=\bigoplus_{S=S_{\min }}^{s N} n(N, S) \mathbf{S},
$$

where $S_{\min }=0$ if $s N$ is an integer, and $S_{\min }=\frac{1}{2}$ if $s N$ is a half-odd integer. The multiplicity of the spin- $S$ representation, which we denote by $n(N, S)$, is computed in the Appendix of [29] ${ }^{1}$

$$
n(N, S)=b(N, S)-b(N, S+1),
$$

where

$$
b(N, r)=\sum_{k=0}^{N}(-1)^{k}\left(\begin{array}{l}
N \\
k
\end{array}\right)\left(\begin{array}{c}
(s+1) N+r-(2 s+1) k-1 \\
s N+r-(2 s+1) k
\end{array}\right),
$$

and the sum is restricted so that $s N+r-(2 s+1) k \geq 0$. In view of (2.1), it is necessary that

$$
\sum_{S=S_{\min }}^{s N}(2 S+1) n(N, S)=(2 s+1)^{N} .
$$

Since $M=s N-S$ (1.8) and the values of $S$ range from $S_{\min }$ to $s N$, it follows that the values of $M$ range from 0 to [sN], as anticipated in (1.5). If we naively assume that every solution of the Bethe equations with pairwise distinct roots produces, via (1.6), an su(2) highestweight eigenstate of the Hamiltonian, then the number of solutions of the Bethe equations

${ }^{1}$ This result is derived using an expression for $C_{m}(n, k)$ defined by [30]

$$
\left(1+x+x^{2}+\cdots+x^{m-1}\right)^{n}=\sum_{k=0}^{(m-1) n} C_{m}(n, k) x^{k} .
$$

However, there is a misprint in Eq. (3) of [30]; the correct result is

$$
C_{m}(n, k)=\sum_{j=0}^{n}{ }^{\prime}(-1)^{j}\left(\begin{array}{c}
n \\
j
\end{array}\right)\left(\begin{array}{c}
n-1+k-m j \\
k-m j
\end{array}\right),
$$

where the sum is restricted so that $k-m j \geq 0$. An alternative expression for the multiplicities is given by Lemma 6 in [11. 
with $M$ pairwise distinct roots should be given by the multiplicity of representations with spin $S=s N-M$. That is,

$$
\mathcal{N}(N, M) \stackrel{?}{=} n(N, s N-M) .
$$

A proof of (2.5) (based on the string hypothesis [1, 9, 10], which requires $N \rightarrow \infty$ ) was proposed in [11]. Related arguments were given in [5, 6].

This naive conjecture is wrong for two reasons. First, as discussed in detail in Appendix A, there exist unphysical singular solutions with pairwise distinct roots, which do not produce eigenstates of the Hamiltonian. Second, as discussed in Appendix B, there exist "strange" solutions with repeated roots, which do produce eigenstates of the Hamiltonian. Correcting for these two effects, we arrive at the following conjecture:

$$
\mathcal{N}(N, M)-\mathcal{N}_{s}(N, M)+\mathcal{N}_{s p}(N, M)+\mathcal{N}_{\text {strange }}(N, M)=n(N, s N-M),
$$

where $\mathcal{N}_{s}(N, M)$ is the number of solutions of the Bethe equations (1.5) with pairwise distinct roots that are singular (i.e., that contain (1.9)); and $\mathcal{N}_{s p}(N, M)$ is the number of such singular solutions that satisfy (A.19)

$$
\left[(-1)^{2 s} \prod_{k=2 s+2}^{M}\left(\frac{\lambda_{k}+i s}{\lambda_{k}-i s}\right)\right]^{N}=1,
$$

and hence are physical. (As explained in Appendix A, singular solutions require regularization; and the requirement that the corresponding Bethe states be eigenstates of the Hamiltonian leads to generalized Bethe equations, which in turn lead to the constraint (2.7). This constraint reduces for $s=1 / 2$ to the one recently found in [25].) Note that $\mathcal{N}_{s}-\mathcal{N}_{s p}$ is the number of unphysical singular solutions. Finally, $\mathcal{N}_{\text {strange }}(N, M)$ is the number of strange solutions, whose roots are not pairwise distinct but which nevertheless produce eigenstates of the Hamiltonian.

In principle, there are many possible types of solutions with repeated roots; and each type should have its own physicality condition. Therefore, in practice, it is difficult to know if one has found all the strange solutions. This, in turn, makes the conjecture (2.6) difficult to check. However, since $\mathcal{N}_{\text {strange }}(N, M) \geq 0$, this conjecture implies the inequality

$$
\mathcal{N}(N, M)-\mathcal{N}_{s}(N, M)+\mathcal{N}_{s p}(N, M) \leq n(N, s N-M),
$$

which is much easier to check. Its physical meaning is that the Bethe equations (1.5) generally have too many solutions with pairwise distinct roots; but after discarding the unphysical singular solutions, there may not remain sufficiently many solutions with pairwise distinct roots to account for all $(2 s+1)^{N}$ eigenstates of the Hamiltonian (1.1). The equality (2.6) further asserts that any such deficit is made up by certain solutions with repeated roots.

\section{$3 \quad$ Numerical results}

Using homotopy continuation with Bertini [28] (see [26] and references therein for further details), we have solved the Bethe equations (1.5) numerically for $s=1$ and $s=3 / 2$ with 
$N=2,3, \ldots, 8$. The results are presented in a set of supplemental tables [31, and are summarized below.

\subsection{The case $s=1$}

The results for $s=1$ are summarized in Table 1. For each set of values $(N, M)$, we report a set of five integers:

$$
\left(\mathcal{N}, \mathcal{N}_{s}, \mathcal{N}_{s p}, \mathcal{N}_{\text {strange }} ; \mathcal{N}-\mathcal{N}_{s}+\mathcal{N}_{\text {sp }}+\mathcal{N}_{\text {strange }}\right)
$$

where $\mathcal{N}$ is the number of solutions of the Bethe equations with pairwise distinct roots; $\mathcal{N}_{s}$ is the number of singular solutions (i.e., that contain $\pm i, 0$ ) with pairwise distinct roots; $\mathcal{N}_{s p}$ is the number of such singular solutions that are physical (i.e., that satisfy (2.7) with $s=1$ ); and $\mathcal{N}_{\text {strange }}$ is the number of solutions with repeated roots that are physical. The quantities $\mathcal{N}-\mathcal{N}_{s}+\mathcal{N}_{s p}+\mathcal{N}_{\text {strange }}$ in all the entries of Table 1 coincide with $n(N, N-M)$, in perfect agreement with the conjecture (2.6).

\begin{tabular}{|c|c|c|c|c|c|c|c|c|}
\hline$M$ & 1 & 2 & 3 & 4 & 5 & 6 & 7 & 8 \\
\hline 2 & $(1,0,0,0 ; 1)$ & $(1,0,0,0 ; 1)$ & & & & & & \\
\hline 3 & $(2,0,0,0 ; 2)$ & $(3,0,0,0 ; 3)$ & $(1,1,1,0 ; 1)$ & & & & & \\
\hline 4 & $(3,0,0,0 ; 3)$ & $(6,0,0,0 ; 6)$ & $(6,1,1,0 ; 6)$ & $(4,2,0,1 ; 3)$ & & & & \\
\hline 5 & $(4,0,0,0 ; 4)$ & $(10,0,0,0 ; 10)$ & $(15,1,1,0 ; 15)$ & $(19,4,0,0 ; 15)$ & $(14,10,2,0 ; 6)$ & & & \\
\hline 6 & $(5,0,0,0 ; 5)$ & $(15,0,0,0 ; 15)$ & $(29,1,1,0 ; 29)$ & $(43,4,0,1 ; 40)$ & $(48,15,3,0 ; 36)$ & $(41,28,0,2 ; 15)$ & & \\
\hline 7 & $(6,0,0,0 ; 6)$ & $(21,0,0,0 ; 21)$ & $(49,1,1,0 ; 49)$ & $(90,6,0,0 ; 84)$ & $(123,21,3,0 ; 105)$ & $(141,50,0,0 ; 91)$ & $(120,90,6,0 ; 36)$ & \\
\hline 8 & $(7,0,0,0 ; 7)$ & $(28,0,0,0 ; 28)$ & $(76,1,1,0 ; 76)$ & $(159,6,0,1 ; 154)$ & $(262,28,4,0 ; 238)$ & $(351,74,0,3 ; 280)$ & $(384,161,9,0 ; 232)$ & $(345,260,0,6 ; 91)$ \\
\hline
\end{tabular}

Table 1: The values $\left(\mathcal{N}, \mathcal{N}_{s}, \mathcal{N}_{s p}, \mathcal{N}_{\text {strange }} ; \mathcal{N}-\mathcal{N}_{s}+\mathcal{N}_{s p}+\mathcal{N}_{\text {strange }}\right)$ for $s=1$ and given values of $N$ and $M$.

We observe that, up to $N=8$, all the strange solutions contain $\pm i, 0,0$; and the remaining $M-4$ roots satisfy (B.16)

$$
\prod_{j=5}^{M}\left(\frac{\lambda_{j}+2 i}{\lambda_{j}-2 i}\right)=(-1)^{N},
$$

which is a condition for singular solutions of this particular type to be physical. These solutions are listed in Table 2.2 It remains an open question whether there exist additional types of strange solutions for $s=1$ and $N>8$.

\subsection{The case $s=3 / 2$}

The results for $s=3 / 2$ are summarized in Tables 3 and 4, Here the singular solutions contain $\pm 3 i / 2, \pm i / 2$; and the physical singular solutions satisfy (2.7) with $s=3 / 2$. Up to $N=7$, the quantities $\mathcal{N}-\mathcal{N}_{s}+\mathcal{N}_{s p}+\mathcal{N}_{\text {strange }}$ in all the entries of these tables coincide with $n\left(N, \frac{3 N}{2}-M\right)$, in agreement with the conjecture (2.6).

\footnotetext{
${ }^{2}$ In [17, the solution for $N=M=4$ was found (see Eqs. (40), (41)), and the corresponding Bethe vector was asserted to be the first and (at that time) only example with equal roots. The two solutions for $N=M=6$ were also noted in [17] (see Eq. (20)), but were derived using the erroneous Eq. (19). See also footnote 4
} 


\begin{tabular}{|c|c|c|}
\hline $\mathrm{N}$ & $\mathrm{M}$ & $\lambda_{1}, \ldots, \lambda_{M}$ \\
\hline 4 & 4 & $\pm i, 0,0$ \\
\hline 6 & 4 & $\pm i, 0,0$ \\
\hline 6 & 6 & $\pm i, 0,0, \pm 0.474498 i$ \\
6 & 6 & $\pm i, 0,0, \pm 2.10749 i$ \\
\hline 8 & 4 & $\pm i, 0,0$ \\
\hline 8 & 6 & $\pm i, 0,0, \pm 0.497637 i$ \\
8 & 6 & $\pm i, 0,0, \pm 2.0095 i$ \\
8 & 6 & $\pm i, 0,0, \pm 1$ \\
\hline 8 & 8 & $\pm i, 0,0, \pm 0.833228, \pm 2.06462 i$ \\
8 & 8 & $0,0, \pm 0.500018 i, \pm 1.49494 i$ \\
8 & 8 & $0.15263 \pm 0.474611 i,-0.15263 \pm 0.474611 i$ \\
8 & 8 & $\pm i, 0,0,0.00281 i, \pm 3.53743 i$ \\
8 & 8 & $\pm i, 0,0,0.594869 \pm 0.532284 i,-0.594869 \pm 0.532284 i$ \\
8 & 8 & $\pm i, 0,0, \pm 0.498746 i, \pm 2.13944 i$ \\
\hline
\end{tabular}

Table 2: Strange solutions for $s=1$ up to $N=8$.

Our results for $N=8$ and $M=6,8,10,12$ are inconclusive. In order to have agreement with the conjecture (2.6), a small number of strange solutions (as indicated by the entries marked with "?" in Tables 3 and 4) should exist for those cases. Unfortunately, we have not managed to identify those solutions. We speculate that those missing solutions are strange singular solutions of a new type, containing both an exact 4 -string $( \pm 3 i / 2, \pm i / 2)$ and an exact 2 -string $( \pm i / 2)$. However, as discussed in Section B.2.2, we have not been able to derive the consistency condition(s) for such solutions (i.e., the conditions for the corresponding Bethe states to be eigenstates of the Hamiltonian). Hence, we can neither confirm nor contradict the conjecture for $N=8$ and $M=6,8,10,12$. The inequality (2.8) is nevertheless satisfied for these cases.

\begin{tabular}{|l|c|c|c|c|c|c|}
\hline$M$ & 1 & 2 & 3 & 4 & 5 & 6 \\
\hline 2 & $(1,0,0,0 ; 1)$ & $(1,0,0,0 ; 1)$ & $(1,0,0,0 ; 1)$ & & \\
3 & $(2,0,0,0 ; 2)$ & $(2,0,0,1 ; 3)$ & $(4,0,0,0 ; 4)$ & $(3,1,0,0 ; 2)$ & & $(8,6,2,0 ; 4)$ \\
4 & $(3,0,0,0 ; 3)$ & $(6,0,0,0 ; 6)$ & $(10,0,0,0 ; 10)$ & $(11,1,1,0 ; 11)$ & $(11,3,1,0 ; 9)$ & $(44,10,0,0 ; 34)$ \\
5 & $(4,0,0,0 ; 4)$ & $(10,0,0,0 ; 10)$ & $(20,0,0,0 ; 20)$ & $(31,1,0,0 ; 30)$ & $(40,4,0,0 ; 36)$ & $(132,15,3,0 ; 120)$ \\
6 & $(5,0,0,0 ; 5)$ & $(13,0,0,2 ; 15)$ & $(35,0,0,0 ; 35)$ & $(64,1,1,0 ; 64)$ & $(100,5,1,0 ; 96)$ & $(336,21,0,0 ; 315)$ \\
7 & $(6,0,0,0 ; 6)$ & $(21,0,0,0 ; 21)$ & $(56,0,0,0 ; 56)$ & $(120,1,0,0 ; 119)$ & $(216,6,0,0 ; 210)$ & $(723,28,4,0[1 ? ; 699)$ \\
8 & $(7,0,0,0 ; 7)$ & $(28,0,0,0 ; 28)$ & $(84,0,0,0 ; 84)$ & $(202,1,1,0 ; 202)$ & $(412,7,1,0 ; 406)$ & $(723,699)$ \\
\hline
\end{tabular}

Table 3: The values $\left(\mathcal{N}, \mathcal{N}_{s}, \mathcal{N}_{s p}, \mathcal{N}_{\text {strange }} ; \mathcal{N}-\mathcal{N}_{s}+\mathcal{N}_{s p}+\mathcal{N}_{\text {strange }}\right)$ for $s=3 / 2$ and given values of $N$ and $M$ (part 1).

\begin{tabular}{|c|c|c|c|c|c|c|}
\hline$M$ & 7 & 8 & 9 & 10 & 11 \\
\hline 2 & & & & & & \\
3 & & & & & \\
4 & $(40,20,0,0 ; 20)$ & & & & \\
5 & $(152,35,3,0 ; 120)$ & $(150,65,5,0 ; 90)$ & $(130,101,5,0 ; 34)$ & & \\
6 & $(456,56,0,0 ; 400)$ & $(545,119,0,0 ; 426)$ & $(580,216,0,0 ; 364)$ & $(543,333,0,0 ; 210)$ & & \\
7 & $(1124,84,4,0 ; 1044)$ & $(1544,203,9,0[1 ?] ; 1350)$ & $(1909,413,9,0 ; 1505)$ & $(2107,728,16,0[5 ?] ; 1395)$ & $(2112,1128,16,0 ; 1000)$ & $(1893,1554,22,0[3 ?] ; 361)$ \\
\hline
\end{tabular}

Table 4: The values $\left(\mathcal{N}, \mathcal{N}_{s}, \mathcal{N}_{s p}, \mathcal{N}_{\text {strange }} ; \mathcal{N}-\mathcal{N}_{s}+\mathcal{N}_{s p}+\mathcal{N}_{\text {strange }}\right)$ for $s=3 / 2$ and given values of $N$ and $M$ (part 2). 
We have found strange solutions for $N=3,6$ with $M=2$, which are listed in Table 5 , These solutions are not singular, and therefore obey the consistency conditions for strange non-singular solutions discussed in Sec. B.1, namely (B.11) with $k=2$. For the $M=2$ case considered here, these equations reduce to

$$
\left(\frac{\mu-i s}{\mu+i s}\right)^{N}=-1, \quad \frac{\partial}{\partial \mu}\left(\frac{\mu-i s}{\mu+i s}\right)^{N}=-4 i .
$$

The solution $\{0,0\}$ for $s=3 / 2, N=3$ is particularly noteworthy, as it is surely the simplest physical solution of the Bethe equations with repeated roots.

\begin{tabular}{|c|c|c|}
\hline $\mathrm{N}$ & $\mathrm{M}$ & $\lambda_{1}, \ldots, \lambda_{M}$ \\
\hline 3 & 2 & 0,0 \\
\hline 6 & 2 & $1.5,1.5$ \\
6 & 2 & $-1.5,-1.5$ \\
\hline
\end{tabular}

Table 5: Strange solutions for $s=3 / 2$ up to $N=7$.

\section{Discussion}

Previous studies [5, 6, 11] of the completeness of the solutions of the spin- $s$ Bethe equations (1.5) have relied on the string hypothesis [1, 9, 10], which requires $N \rightarrow \infty$ and whose status remains unclear (see below).

We have initiated here instead an ab initio investigation of the completeness problem. To this end, we have formulated a conjecture (2.6) for the number of solutions with pairwise distinct roots in terms of the numbers of singular and strange solutions. We have tested this conjecture for small values of $s$ and $N$. For $s=1$, there is perfect agreement with the conjecture up to $N=8$ (i.e., as far as we have checked). For $s=3 / 2$, there is perfect agreement with the conjecture up to $N=7$. However, for $N=8$ and $M=6,8,10,12$, our results are inconclusive. We expect that the conjecture will continue to hold for these cases, and that the observed discrepancies will be resolved by the existence of a new type of strange singular solution, which remains to be confirmed. The problem of dealing with new types of strange solutions will likely grow for larger values of $s$ and $N$. However, one can hope that a pattern for the types of strange solutions (for given values of $s$ and $N$ ) will eventually emerge.

We have exhibited some strange solutions in Tables 2 and 5 . Especially notable is the solution $\{0,0\}$ for $s=3 / 2, N=3$, which is probably the simplest example of a solution with equal roots that corresponds to an eigenstate of the Hamiltonian.

Several patterns can be discerned from Tables 1, 3, 4, similarly to the $s=1 / 2$ case [26]. In particular, for $M \sim s N$ and most values of $N$, the number of unphysical singular solutions $\mathcal{N}_{s}-\mathcal{N}_{s p}$ is comparable to $n(N, s N-M)$. This suggests that the naive formula (2.5) for the number of solutions of the Bethe equations is incorrect not only for small values of $N$, but also for $N \rightarrow \infty$. This calls into question the proofs of (2.5). The related question [26] 
of how singular solutions fit within the string hypothesis also remains to be clarified. It is tempting to speculate that the number of strange solutions in the spin-0 sector $(M=s N)$ grows sufficiently fast in the thermodynamic limit to account for the still-mysterious RSOS structure [8] of the antiferromagnetic ground state.

\section{Acknowledgments}

WH's research has been supported by the Mathematical Biosciences Institute and the National Science Foundation under Grant DMS 0931642. The work of RN was supported in part by the National Science Foundation under Grant PHY-1212337, and by a Cooper fellowship. WH and AS also thank DARPA/AFRL Grant G-2457-2 for supporting their work.

\section{A Singular solutions}

We recall that the spin-s Bethe equations (1.5) admit solutions containing

$$
\{i s, i(s-1), \ldots,-i(s-1),-i s\}
$$

which is a so-called exact string of length $2 s+1$ centered at the origin. Since both the corresponding energy (1.4) and (with our normalization of the $R$-matrix, see (A.2) below) the corresponding Bethe state (1.6) are singular, we call such solutions "singular". It is always possible to regularize such solutions in a way that the corresponding energies and Bethe states are finite in the limit that the regulator is removed. However, the states obtained in this way are not guaranteed to be eigenstates of the Hamiltonian. We term "physical" a singular solution that leads in this way to an eigenstate of the Hamiltonian; otherwise, we term the singular solution "unphysical". Generally, only a small fraction of the singular solutions are physical.

We derive in this section a simple criterion for identifying the physical singular solutions. We proceed by generalizing the $s=1 / 2$ analysis in [25] to general values of $s$.

We use the standard $\left(\frac{1}{2}, s\right)$ solution of the Yang-Baxter equation (see e.g. [6])

$$
R^{\left(\frac{1}{2}, s\right)}(\lambda)=\frac{1}{\lambda+i s}(\lambda+i \vec{\sigma} \dot{\otimes} \vec{s})
$$

except with an unusual normalization, such that the $R$-matrix is singular at $\lambda=-i s$. As in the Hamiltonian (1.1), $\vec{s}$ are the spin-s generators of $s u(2)$; and $\vec{\sigma}$ are the standard Pauli matrices. The monodromy matrix is given by

$$
T_{a}(\lambda)=R_{a N}^{\left(\frac{1}{2}, s\right)}(\lambda) \cdots R_{a 1}^{\left(\frac{1}{2}, s\right)}(\lambda)=\left(\begin{array}{cc}
A(\lambda) & B(\lambda) \\
C(\lambda) & D(\lambda)
\end{array}\right)
$$

and the transfer matrix is given by

$$
t(\lambda)=\operatorname{tr}_{a} T_{a}(\lambda)=A(\lambda)+D(\lambda) .
$$


Its action on an off-shell Bethe vector (1.6) is given by [1]

$$
t(\lambda)\left|\lambda_{1}, \ldots, \lambda_{M}\right\rangle=\Lambda(\lambda,\{\lambda\})\left|\lambda_{1}, \ldots, \lambda_{M}\right\rangle+\sum_{k=1}^{M} F_{k}(\lambda,\{\lambda\})\left|v_{k}(\lambda,\{\lambda\})\right\rangle
$$

where

$$
\left|v_{k}(\lambda,\{\lambda\})\right\rangle \equiv B(\lambda) \prod_{\substack{j \neq k \\ j=1}}^{M} B\left(\lambda_{j}\right)|0\rangle
$$

and

$$
\begin{aligned}
\Lambda(\lambda,\{\lambda\}) & =\prod_{j=1}^{M} \frac{\lambda-\lambda_{j}-i}{\lambda-\lambda_{j}}+\left(\frac{\lambda-i s}{\lambda+i s}\right)^{N} \prod_{j=1}^{M} \frac{\lambda-\lambda_{j}+i}{\lambda-\lambda_{j}} \\
F_{k}(\lambda,\{\lambda\}) & =\frac{i}{\lambda-\lambda_{k}}\left[\prod_{j \neq k}^{M} \frac{\lambda_{k}-\lambda_{j}-i}{\lambda_{k}-\lambda_{j}}-\left(\frac{\lambda_{k}-i s}{\lambda_{k}+i s}\right)^{N} \prod_{j \neq k}^{M} \frac{\lambda_{k}-\lambda_{j}+i}{\lambda_{k}-\lambda_{j}}\right] .
\end{aligned}
$$

If the set $\left\{\lambda_{1}, \ldots, \lambda_{M}\right\}$ is not singular (i.e., it does not contain the subset (A.1) ), then the conditions

$$
F_{k}(\lambda,\{\lambda\})=0, \quad k=1, \ldots, M
$$

imply that the "unwanted" terms in (A.5) drop out; and hence the vector $\left|\lambda_{1}, \ldots, \lambda_{M}\right\rangle$ is an eigenvector of the transfer matrix, with eigenvalue (A.7). Eqs. (A.9) are equivalent to the Bethe equations (1.5).

We henceforth consider a solution $\left\{\lambda_{1}, \ldots, \lambda_{M}\right\}$ (where $M \geq 2 s+1$ ) of the Bethe equations (1.5) that is singular, i.e. it does contain the subset (A.1). For definiteness, we order the roots such that the first $2 s+1$ roots are given by (A.1), respectively; that is,

$$
\lambda_{j}=i(s+1-j), \quad j=1, \ldots, 2 s+1 .
$$

We furthermore assume that there are no repeated roots. (Cases with repeated roots are considered in Section B.2.) That is, the remaining roots $\lambda_{2 s+2}, \ldots, \lambda_{M}$ are distinct and are not equal to $i s, i(s-1), \ldots,-i(s-1),-i s$. We regularize this solution as follows

$$
\lambda_{j}=i(s+1-j)+\epsilon+c_{j} \epsilon^{N}, \quad j=1, \ldots, 2 s+1,
$$

where $\epsilon$ is the regulator, and $c_{1}, \ldots, c_{2 s+1}$ are constants (independent of $\epsilon$ ) that are still to be determined. The key observation is that, due to our normalization of the $R$-matrix (A.2), $B\left(\lambda_{2 s+1}\right)$ is singular

$$
B\left(\lambda_{2 s+1}\right) \sim \frac{1}{\epsilon^{N}} ; \quad B\left(\lambda_{k}\right) \sim 1, \quad k=1, \ldots, 2 s
$$


as $\epsilon \rightarrow 0$. Hence, in view of (A.5), the vector $\left|\lambda_{1}, \ldots, \lambda_{M}\right\rangle$ is an eigenvector of the transfer matrix if 3

$$
F_{k}(\lambda,\{\lambda\}) \sim \epsilon^{N+1}, \quad k=1, \ldots, 2 s ; \quad F_{2 s+1}(\lambda,\{\lambda\}) \sim \epsilon
$$

as $\epsilon \rightarrow 0$, together with

$$
F_{k}(\lambda,\{\lambda\})=0, \quad k=2 s+2, \ldots, M .
$$

We call (A.13) generalized Bethe equations, since they are evidently generalizations of (A.9).

Evaluating $F_{k}(\lambda,\{\lambda\})$ using (A.8) and (A.11), the generalized Bethe equations (A.13) imply

$$
\begin{aligned}
c_{1}-c_{2} & =\frac{(2 s+1)}{(2 i s)^{N-1}} \prod_{j=2 s+2}^{M} \frac{\lambda_{j}-i(s+1)}{\lambda_{j}-i(s-1)} \\
c_{k}-c_{k+1} & =-\left(c_{k-1}-c_{k}\right)\left(\frac{1-k}{2 s+1-k}\right)^{N-1}\left(\frac{2 s+2-k}{k}\right) \prod_{j=2 s+2}^{M} \frac{\lambda_{j}-i(s+2-k)}{\lambda_{j}-i(s-k)}, k=2, \ldots, 2 s, \\
c_{2 s}-c_{2 s+1} & =-\frac{(2 s+1)}{(-2 i s)^{N-1}} \prod_{j=2 s+2}^{M} \frac{\lambda_{j}+i(s+1)}{\lambda_{j}+i(s-1)} .
\end{aligned}
$$

Forming the product of the first $2 s$ equations in (A.15), we obtain an expression for $c_{2 s}-c_{2 s+1}$, which is consistent with the final equation in (A.15) provided that

$$
\prod_{j=2 s+2}^{M}\left(\frac{\lambda_{j}-i s}{\lambda_{j}+i s}\right)\left(\frac{\lambda_{j}-i(s+1)}{\lambda_{j}+i(s+1)}\right)=(-1)^{2 s N} \text {. }
$$

The remaining roots $\lambda_{2 s+2}, \ldots, \lambda_{M}$ do not need regularization. They obey the usual Bethe equations (A.14), which imply

$$
\left(\frac{\lambda_{k}+i s}{\lambda_{k}-i s}\right)^{N-1}\left(\frac{\lambda_{k}-i(s+1)}{\lambda_{k}+i(s+1)}\right)=\prod_{\substack{j \neq k \\ j=2 s+2}}^{M} \frac{\lambda_{k}-\lambda_{j}+i}{\lambda_{k}-\lambda_{j}-i}, \quad k=2 s+2, \ldots, M .
$$

Forming the product of these equations, we obtain

$$
\prod_{k=2 s+2}^{M}\left(\frac{\lambda_{k}+i s}{\lambda_{k}-i s}\right)^{N-1}\left(\frac{\lambda_{k}-i(s+1)}{\lambda_{k}+i(s+1)}\right)=1 .
$$

Combining the results (A.16) and (A.18), we arrive at the constraint

$$
\left[(-1)^{2 s} \prod_{k=2 s+2}^{M}\left(\frac{\lambda_{k}+i s}{\lambda_{k}-i s}\right)\right]^{N}=1
$$

This is the condition for the singular solution (A.10) with all roots distinct to be physical. For the case $s=1 / 2$, this equation reduces to the one found in [25].

\footnotetext{
${ }^{3}$ The vector $\lim _{\epsilon \rightarrow 0} \prod_{k=1}^{2 s+1} B\left(\lambda_{k}\right)|0\rangle$ is finite, as shown for the case $s=1 / 2$ in [25].
} 


\section{B Strange solutions}

The Bethe equations (1.5) generally admit many solutions with repeated roots. For $s=1 / 2$ and (at least) $N \leq 14$ [26] such solutions do not lead to eigenstates of the Hamiltonian, i.e. such solutions are unphysical. However, for $s>1 / 2$, there exist "strange" solutions of the Bethe equations with repeated roots that do produce eigenstates of the Hamiltonian, i.e. such solutions are physical. Here we discuss simple criteria for determining if a given solution with repeated roots is physical. We consider separately the cases that the solutions are singular.

\section{B.1 Strange non-singular solutions}

We first consider solutions that are not singular (i.e., do not contain (A.1)) and contain a repeated root. In the simplest such case, a root $\mu$ has multiplicity 2 . That is, the solution has the form

$$
\left\{\mu, \mu, \lambda_{3}, \ldots, \lambda_{M}\right\}
$$

where $\mu, \lambda_{3}, \ldots, \lambda_{M}$ are distinct and are not equal to $i s, i(s-1), \ldots,-i(s-1),-i s$. Following [17, 32], one finds that

$$
\begin{aligned}
& t(\lambda)\left|\mu, \mu, \lambda_{3}, \ldots, \lambda_{M}\right\rangle=\Lambda(\lambda, \mu,\{\lambda\})\left|\mu, \mu, \lambda_{3}, \ldots, \lambda_{M}\right\rangle \\
& \quad+\sum_{k=3}^{M} F_{k}(\lambda, \mu,\{\lambda\}) B(\lambda) B(\mu)^{2} \prod_{\substack{j \neq k \\
j=3}}^{M} B\left(\lambda_{j}\right)|0\rangle+G(\lambda, \mu,\{\lambda\}) B(\lambda) B^{\prime}(\mu) \prod_{j=3}^{M} B\left(\lambda_{j}\right)|0\rangle \\
& \quad+H(\lambda, \mu,\{\lambda\}) B(\lambda) B(\mu) \prod_{j=3}^{M} B\left(\lambda_{j}\right)|0\rangle,
\end{aligned}
$$

where $\Lambda(\lambda, \mu,\{\lambda\})$ and $F_{k}(\lambda, \mu,\{\lambda\})$ are given by (A.7) and (A.8) with $\lambda_{1}=\lambda_{2}=\mu$, respectively; and

$$
\begin{aligned}
G(\lambda, \mu,\{\lambda\})= & -\frac{1}{\lambda-\mu}\left[\prod_{j=3}^{M} \frac{\mu-\lambda_{j}-i}{\mu-\lambda_{j}}+\left(\frac{\mu-i s}{\mu+i s}\right)^{N} \prod_{j=3}^{M} \frac{\mu-\lambda_{j}+i}{\mu-\lambda_{j}}\right] . \\
H(\lambda, \mu,\{\lambda\})= & \frac{i}{\lambda-\mu}\left\{\left(2-\frac{i}{\lambda-\mu}\right) \prod_{j=3}^{M} \frac{\mu-\lambda_{j}-i}{\mu-\lambda_{j}}-\left(2+\frac{i}{\lambda-\mu}\right)\left(\frac{\mu-i s}{\mu+i s}\right)^{N} \prod_{j=3}^{M} \frac{\mu-\lambda_{j}+i}{\mu-\lambda_{j}}\right. \\
& \left.-i \frac{\partial}{\partial \mu}\left[\prod_{j=3}^{M} \frac{\mu-\lambda_{j}-i}{\mu-\lambda_{j}}+\left(\frac{\mu-i s}{\mu+i s}\right)^{N} \prod_{j=3}^{M} \frac{\mu-\lambda_{j}+i}{\mu-\lambda_{j}}\right]\right\} .
\end{aligned}
$$

Hence, besides the "usual" Bethe equations

$$
\begin{aligned}
G(\lambda, \mu,\{\lambda\}) & =0, \\
F_{k}(\lambda, \mu,\{\lambda\}) & =0, \quad k=3, \ldots, M,
\end{aligned}
$$


the additional constraint

$$
H(\lambda, \mu,\{\lambda\})=0
$$

is necessary for all the unwanted terms in (B.2) to drop out. Eq. (B.5) implies that the $\lambda$-dependent terms within braces in $H(\lambda, \mu,\{\lambda\})($ B.4 $)$ cancel. Eq. (B.7) can therefore be rewritten as

$$
\left.\frac{\partial}{\partial \lambda}\left[(\lambda-\mu-i)^{2} \prod_{j=3}^{M} \frac{\lambda-\lambda_{j}-i}{\lambda-\lambda_{j}}+(\lambda-\mu+i)^{2}\left(\frac{\lambda-i s}{\lambda+i s}\right)^{N} \prod_{j=3}^{M} \frac{\lambda-\lambda_{j}+i}{\lambda-\lambda_{j}}\right]\right|_{\lambda=\mu}=0
$$

More generally, a root $\mu$ can have multiplicity $k$. That is, the solution has the form

$$
\{\underbrace{\mu, \ldots, \mu}_{k}, \lambda_{k+1}, \ldots, \lambda_{M}\}
$$

where $\mu, \lambda_{k+1}, \ldots, \lambda_{M}$ are distinct and are not equal to $i s, i(s-1), \ldots,-i(s-1),-i s$. In order to derive all the physical constraints, one can proceed as above using the algebraic Bethe ansatz. A simpler way is based on the observation that the Bethe equations (1.5) are the conditions for the transfer matrix eigenvalue (A.7) to be finite at $\lambda=\lambda_{k}$. Similarly, for

a solution of the form (B.9), the transfer matrix eigenvalue (A.7) becomes

$$
\Lambda=\left(\frac{\lambda-\mu-i}{\lambda-\mu}\right)^{k} \prod_{j=k+1}^{M} \frac{\lambda-\lambda_{j}-i}{\lambda-\lambda_{j}}+\left(\frac{\lambda-\mu+i}{\lambda-\mu}\right)^{k}\left(\frac{\lambda-i s}{\lambda+i s}\right)^{N} \prod_{j=k+1}^{M} \frac{\lambda-\lambda_{j}+i}{\lambda-\lambda_{j}}
$$

Demanding that $\Lambda$ be finite at $\lambda=\mu$ leads to the following constraints

$$
\begin{gathered}
\left.\frac{\partial^{l}}{\partial \lambda^{l}}\left[(\lambda-\mu-i)^{k} \prod_{j=k+1}^{M} \frac{\lambda-\lambda_{j}-i}{\lambda-\lambda_{j}}+(\lambda-\mu+i)^{k}\left(\frac{\lambda-i s}{\lambda+i s}\right)^{N} \prod_{j=k+1}^{M} \frac{\lambda-\lambda_{j}+i}{\lambda-\lambda_{j}}\right]\right|_{\lambda=\mu}=0, \\
l=0,1, \ldots, k-1 .
\end{gathered}
$$

Together with the usual Bethe equations for $\lambda_{k+1}, \ldots, \lambda_{M}$, these are the conditions for the solution (B.9) to be physical. For the case $k=2$, Eqs. (B.11) with $l=0,1$ are equivalent to Eqs. (B.5), (B.8), respectively.

\section{B.2 Strange singular solutions}

We now consider solutions that are singular (i.e., contain (A.1) ) and contain a repeated root. In principle, there are many such possibilities, which should be analyzed individually. For simplicity, we explicitly analyze here only two such cases. 


\section{B.2.1 $s=1$}

We first consider the case that $s=1$ and the root 0 has multiplicity 2 . We order the roots such that the first three are given by (A.1), and the fourth root is the second 0; i.e.

$$
\left(\lambda_{1}, \lambda_{2}, \lambda_{3}, \lambda_{4}\right)=(i, 0,-i, 0) .
$$

We assume that the remaining roots $\lambda_{5}, \ldots, \lambda_{M}$ are distinct and are not equal to $\pm i, 0$; they satisfy the usual Bethe equations (1.5), which reduce in this case to

$$
\left(\frac{\lambda_{k}+i}{\lambda_{k}-i}\right)^{N-2}\left(\frac{\lambda_{k}-2 i}{\lambda_{k}+2 i}\right)=\prod_{\substack{j \neq k \\ j=5}}^{M} \frac{\lambda_{k}-\lambda_{j}+i}{\lambda_{k}-\lambda_{j}-i}, \quad k=5, \ldots, M
$$

We regulate this solution as follows

$$
\begin{aligned}
& \lambda_{1}=i+\epsilon+c_{1} \epsilon^{N}, \\
& \lambda_{2}=\epsilon+c_{2} \epsilon^{\frac{N}{2}}, \\
& \lambda_{3}=-i+\epsilon+c_{3} \epsilon^{N}, \\
& \lambda_{4}=\epsilon+c_{4} \epsilon^{\frac{N}{2}},
\end{aligned}
$$

where $\epsilon$ is the regulator, and $c_{1}, \ldots, c_{4}$ are constants (independent of $\epsilon$ ) that are still to be determined. Note that the repeated roots are regulated with $\epsilon^{\frac{N}{2}}$ instead of $\epsilon^{N}$. Note also that the repeated roots are no longer equal once they are regulated; hence, (B.2) should not be applied.

The generalized Bethe equations (A.13) remain valid for the roots that are not repeated, namely $k=1,3$. They imply

$$
\begin{aligned}
& c_{2} c_{4}=-\frac{12}{(2 i)^{N}} \prod_{j=5}^{M}\left(\frac{\lambda_{j}-2 i}{\lambda_{j}}\right), \\
& c_{2} c_{4}=-\frac{12}{(-2 i)^{N}} \prod_{j=5}^{M}\left(\frac{\lambda_{j}+2 i}{\lambda_{j}}\right),
\end{aligned}
$$

respectively, whose consistency requires

$$
\prod_{j=5}^{M}\left(\frac{\lambda_{j}+2 i}{\lambda_{j}-2 i}\right)=(-1)^{N}
$$

This is the condition for the singular solution (B.12) to be physical 4

\footnotetext{
${ }^{4}$ Solutions of the type (B.12) were already considered in [17] (see Eq. (18)). However, a different physicality condition was proposed there (see Eq. (19)). We believe that the latter is incorrect, since it is based on (B.2), (B.8), which should not be applied to singular solutions.
} 
We note that the generalized Bethe equation (A.13) for the repeated root $(k=2)$ is no longer valid. Indeed, for $\epsilon \rightarrow 0$, we observe that 5

$$
\begin{aligned}
& \left|v_{2}(\lambda,\{\lambda\})\right\rangle=c_{4}|w\rangle+O(1), \\
& \left|v_{4}(\lambda,\{\lambda\})\right\rangle=c_{2}|w\rangle+O(1),
\end{aligned}
$$

where $\left|v_{k}(\lambda,\{\lambda\})\right\rangle$ is defined in (A.6), and $|w\rangle \sim \epsilon^{-N / 2}$. Therefore, the unwanted terms in (A.5) for $k=2$ and $k=4$ are not linearly independent,

$F_{2}(\lambda,\{\lambda\})\left|v_{2}(\lambda,\{\lambda\})\right\rangle+F_{4}(\lambda,\{\lambda\})\left|v_{4}(\lambda,\{\lambda\})\right\rangle=\left[c_{4} F_{2}(\lambda,\{\lambda\})+c_{2} F_{4}(\lambda,\{\lambda\})\right]|w\rangle+O(1)$.

This implies the generalized Bethe equation

$$
c_{4} F_{2}(\lambda,\{\lambda\})+c_{2} F_{4}(\lambda,\{\lambda\}) \sim \epsilon^{\frac{N}{2}+1},
$$

which relates $c_{1}-c_{3}$ to $c_{2} c_{4}$. Imposing the vanishing of the $O(1)$ terms leads to a further constraint.

\section{B.2.2 $s=3 / 2$}

We now consider the case that $s=3 / 2$ and both roots $i / 2$ and $-i / 2$ have multiplicity 2. 6 We order the roots such that

$$
\left(\lambda_{1}, \ldots \lambda_{6}\right)=\left(\frac{3 i}{2}, \frac{i}{2},-\frac{i}{2},-\frac{3 i}{2}, \frac{i}{2},-\frac{i}{2}\right) .
$$

We assume that the remaining roots $\lambda_{7}, \ldots, \lambda_{M}$ are distinct and are not equal to $\pm 3 i / 2, \pm i / 2$; they satisfy the usual Bethe equations (1.5), which reduce in this case to

$$
\left(\frac{\lambda_{k}+\frac{3 i}{2}}{\lambda_{k}-\frac{3 i}{2}}\right)^{N-2}\left(\frac{\lambda_{k}-\frac{5 i}{2}}{\lambda_{k}+\frac{5 i}{2}}\right)\left(\frac{\lambda_{k}-\frac{i}{2}}{\lambda_{k}+\frac{i}{2}}\right)=\prod_{\substack{j \neq k \\ j=7}}^{M} \frac{\lambda_{k}-\lambda_{j}+i}{\lambda_{k}-\lambda_{j}-i}, \quad k=7, \ldots, M
$$

\footnotetext{
${ }^{5}$ We have checked (B.17) explicitly for $N=4$ and $N=6$.

${ }^{6}$ This possibility was considered but rejected (prematurely, in our opinion) in [17] below Eq. (20). As already noted, we believe that (B.11) should not be applied to singular solutions.
} 
Proceeding as in Sec. B.2.1, we regulate this solution as follows

$$
\begin{aligned}
& \lambda_{1}=\frac{3 i}{2}+\epsilon+c_{1} \epsilon^{N}, \\
& \lambda_{2}=\frac{i}{2}+\epsilon+c_{2} \epsilon^{\frac{N}{2}}, \\
& \lambda_{3}=-\frac{i}{2}+\epsilon+c_{3} \epsilon^{\frac{N}{2}}, \\
& \lambda_{4}=-\frac{3 i}{2}+\epsilon+c_{4} \epsilon^{N}, \\
& \lambda_{5}=\frac{i}{2}+\epsilon+c_{5} \epsilon^{\frac{N}{2}}, \\
& \lambda_{6}=-\frac{i}{2}+\epsilon+c_{6} \epsilon^{\frac{N}{2}},
\end{aligned}
$$

where $\epsilon$ is the regulator, and $c_{1}, \ldots, c_{6}$ are constants (independent of $\epsilon$ ) that are still to be determined.

The generalized Bethe equations $F_{1} \sim \epsilon^{N+1}$ and $F_{4} \sim \epsilon$ imply

$$
\begin{aligned}
& c_{2} c_{5}=\frac{8}{(3 i)^{N-2}} \prod_{j=7}^{M}\left(\frac{\lambda_{j}-\frac{5 i}{2}}{\lambda_{j}-\frac{i}{2}}\right), \\
& c_{3} c_{6}=\frac{8}{(-3 i)^{N-2}} \prod_{j=7}^{M}\left(\frac{\lambda_{j}+\frac{5 i}{2}}{\lambda_{j}+\frac{i}{2}}\right),
\end{aligned}
$$

respectively. In contrast with (B.15), these relations do not immediately yield a consistency condition analogous to (B.16). Assuming relations similar to (B.17) for the unwanted terms associated with each set of repeated roots, we obtain

$$
\begin{aligned}
& F_{2}(\lambda,\{\lambda\})\left|v_{2}(\lambda,\{\lambda\})\right\rangle+F_{5}(\lambda,\{\lambda\})\left|v_{5}(\lambda,\{\lambda\})\right\rangle=\left[c_{5} F_{2}(\lambda,\{\lambda\})+c_{2} F_{5}(\lambda,\{\lambda\})\right]|w\rangle+O(1), \\
& F_{3}(\lambda,\{\lambda\})\left|v_{3}(\lambda,\{\lambda\})\right\rangle+F_{6}(\lambda,\{\lambda\})\left|v_{6}(\lambda,\{\lambda\})\right\rangle=\left[c_{6} F_{3}(\lambda,\{\lambda\})+c_{3} F_{6}(\lambda,\{\lambda\})\right]\left|w^{\prime}\right\rangle+O(1),
\end{aligned}
$$

which imply the corresponding constraints

$$
\begin{aligned}
& c_{5} F_{2}(\lambda,\{\lambda\})+c_{2} F_{5}(\lambda,\{\lambda\}) \sim \epsilon^{\frac{N}{2}+1}, \\
& c_{6} F_{3}(\lambda,\{\lambda\})+c_{3} F_{6}(\lambda,\{\lambda\}) \sim \epsilon^{\frac{N}{2}+1} .
\end{aligned}
$$

These constraints lead to expressions for $c_{1}$ and $c_{4}$ in terms of $c_{2} c_{5}$ and $c_{3} c_{6}$.

In order to derive the sought-after consistency condition, we need a relation between $c_{2} c_{5}$ and $c_{3} c_{6}$ in $(\overline{B .23})$, which presumably can be obtained by imposing the vanishing of the $O(1)$ terms in (B.24). Unfortunately, a direct computation of these terms for the first nontrivial case $(N=8)$ is out of reach. 


\section{References}

[1] L. D. Faddeev, "How algebraic Bethe ansatz works for integrable models," in Symétries Quantiques (Les Houches Summer School Proceedings vol 64), A. Connes, K. Gawedzki, and J. Zinn-Justin, eds., pp. 149-219. North Holland, 1998. arXiv:hep-th/9605187 [hep-th].

[2] A. Zamolodchikov and V. Fateev, "Model factorized S matrix and an integrable Heisenberg chain with spin 1," Sov.J.Nucl.Phys. 32 (1980) 298-303.

[3] P. Kulish, N. Y. Reshetikhin, and E. Sklyanin, "Yang-Baxter Equation and Representation Theory. 1.," Lett.Math.Phys. 5 (1981) 393-403.

[4] P. Kulish and E. Sklyanin, "Quantum spectral transform method. Recent developments," Lect.Notes Phys. 151 (1982) 61-119.

[5] L. Takhtajan, "The picture of low-lying excitations in the isotropic Heisenberg chain of arbitrary spins," Phys.Lett. A87 (1982) 479-482.

[6] H. M. Babujian, "Exact solution of the isotropic Heisenberg chain with arbitrary spins: thermodynamics of the model," Nucl.Phys. B215 (1983) 317-336.

[7] I. Affleck, D. Gepner, H. Schulz, and T. Ziman, "Critical behavior of spin s Heisenberg antiferromagnetic chains: analytic and numerical results," J.Phys. A22 (1989) 511.

[8] N. Reshetikhin, "S matrices in integrable models of isotropical magnetic chains. 1.," J.Phys. A24 (1991) 3299-3310.

[9] H. Bethe, "On the theory of metals. 1. Eigenvalues and eigenfunctions for the linear atomic chain," Z.Phys. 71 (1931) 205-226.

[10] M. Takahashi, "One-dimensional Heisenberg model at finite temperature," Prog.Theor.Phys. 46 (1971) 401-415.

[11] A. N. Kirillov, "Combinatorial identities, and completeness of eigenstates for the Heisenberg magnet," J. Sov. Math. 30 (1985) 2298.

[12] R. P. Langlands and Y. Saint-Aubin, "Algebro-geometric aspects of the Bethe equations," in Strings and symmetries (Istanbul, 1994), vol. 447 of Lecture Notes in Phys., pp. 40-53. Springer, Berlin, 1995.

[13] R. P. Langlands and Y. Saint-Aubin, "Aspects combinatoires des équations de Bethe," in Advances in mathematical sciences: CRM's 25 years (Montreal, PQ, 1994), L. Vinet, ed., vol. 11 of CRM Proc. Lecture Notes, pp. 231-301. Amer. Math. Soc., Providence, RI, 1997.

[14] K. Fabricius and B. M. McCoy, "Bethe's equation is incomplete for the XXZ model at roots of unity," J.Statist.Phys. 103 (2001) 647-678, arXiv:cond-mat/0009279 [cond-mat.stat-mech]. 
[15] R. J. Baxter, "Completeness of the Bethe ansatz for the six and eight vertex models," J.Statist.Phys. 108 (2002) 1-48, arXiv: cond-mat/0111188 [cond-mat].

[16] E. Mukhin, V. Tarasov, and A. Varchenko, "Bethe algebra of homogeneous XXX Heisenberg model has simple spectrum," Commun. Math. Phys. 288 (2009) 1-42, arXiv:0706.0688 [math].

[17] L. V. Avdeev and A. A. Vladimirov, "Exceptional solutions of the Bethe ansatz equations," Theor. Math. Phys. 69 (1987) 1071.

[18] F. H. L. Essler, V. E. Korepin, and K. Schoutens, "Fine structure of the Bethe ansatz for the spin 1/2 Heisenberg XXX model," J.Phys. A25 (1992) 4115-4126.

[19] R. Siddharthan, "Singularities in the Bethe solution of the XXX and XXZ Heisenberg spin chains," arXiv: cond-mat/9804210 [cond-mat].

[20] J. D. Noh, D.-S. Lee, and D. Kim, "Origin of the singular Bethe ansatz solutions for the Heisenberg XXZ spin chain," Physica A 287 (2000) 167.

[21] N. Beisert, J. A. Minahan, M. Staudacher, and K. Zarembo, "Stringing spins and spinning strings," JHEP 0309 (2003) 010, arXiv:hep-th/0306139 [hep-th].

[22] N. Beisert, V. Dippel, and M. Staudacher, "A Novel long range spin chain and planar N=4 super Yang-Mills," JHEP 0407 (2004) 075, arXiv: hep-th/0405001 [hep-th].

[23] R. Hagemans and J.-S. Caux, "Deformed strings in the Heisenberg model," J.Phys.A 40 (2007) 14605, arXiv:0707.2803 [cond-mat].

[24] G. Arutyunov, S. Frolov, and A. Sfondrini, "Exceptional Operators in N=4 super Yang-Mills," JHEP 1209 (2012) 006, arXiv:1205.6660 [hep-th].

[25] R. I. Nepomechie and C. Wang, "Algebraic Bethe ansatz for singular solutions," J.Phys. A46 (2013) 325002, arXiv:1304.7978 [hep-th].

[26] W. Hao, R. I. Nepomechie, and A. J. Sommese, "Completeness of solutions of Bethe's equations," Phys.Rev.E 88 (2013) 052113, arXiv:1308.4645 [math-ph].

[27] A. J. Sommese and C. W. Wampler, The Numerical Solution Of Systems Of Polynomials Arising In Engineering And Science. World Scientific Publishing Company Incorporated, 2005.

[28] D. J. Bates, J. D. Hauenstein, A. J. Sommese, and C. W. Wampler, Numerically Solving Polynomial Systems with Bertini. SIAM, 2013.

[29] J. R. G. Mendonça, "Exact eigenspectrum of the symmetric simple exclusion process on the complete, complete bipartite, and related graphs," J.Phys. A46 (2013) 295001, arXiv:1207.4106 [cond-mat.stat-mech].

[30] R. C. Bollinger, "Extended Pascal triangles," Mathematics Magazine 66 (1993) 87-94. 
[31] See associated supplemental material on the arxiv.

[32] A. Izergin and V. Korepin, "Pauli principle for one-dimensional bosons and the algebraic Bethe ansatz," Lett.Math.Phys. 6 (1982) 283-288. 